
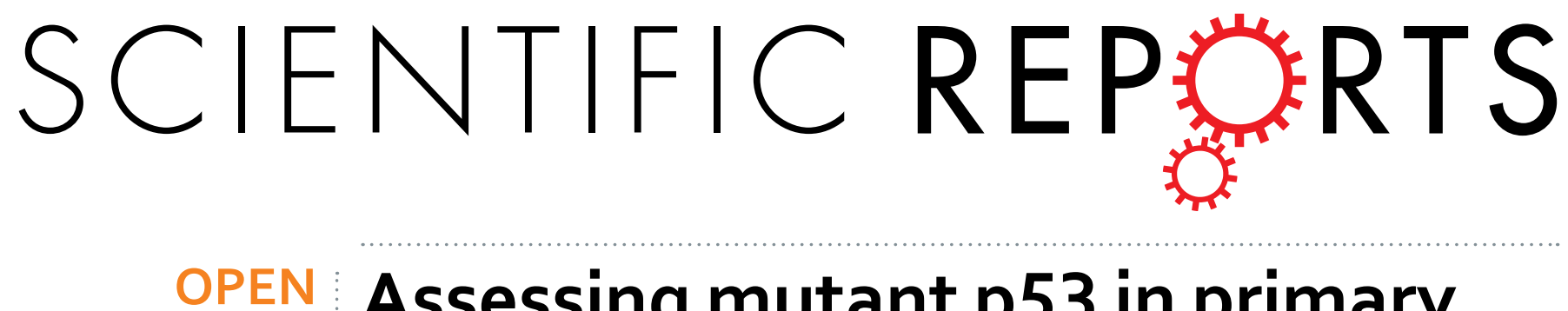

\title{
Assessing mutant p53 in primary high-grade serous ovarian cancer using immunohistochemistry and massively parallel sequencing
}

Accepted: 27 April 2016

Published: 18 May 2016
Alexander J. Cole ${ }^{1}$, Trisha Dwight ${ }^{1}$, Anthony J. Gill ${ }^{2}$, Kristie-Ann Dickson ${ }^{1}$, Ying Zhu ${ }^{3}$, Adele Clarkson ${ }^{2}$, Gregory B. Gard ${ }^{4}$, Jayne Maidens ${ }^{4}$, Susan Valmadre ${ }^{5}$, Roderick Clifton-Bligh ${ }^{1}$ \& Deborah J. Marsh ${ }^{1}$

The tumour suppressor $\mathrm{p} 53$ is mutated in cancer, including over $96 \%$ of high-grade serous ovarian cancer (HGSOC). Mutations cause loss of wild-type $\mathrm{p} 53$ function due to either gain of abnormal function of mutant p53 (mutp53), or absent to low mutp53. Massively parallel sequencing (MPS) enables increased accuracy of detection of somatic variants in heterogeneous tumours. We used MPS and immunohistochemistry (IHC) to characterise HGSOCs for TP53 mutation and p53 expression. TP53 mutation was identified in $94 \%(68 / 72)$ of HGSOCs, $62 \%$ of which were missense. Missense mutations demonstrated high $\mathrm{p} 53$ by IHC, as did $35 \%$ (9/26) of non-missense mutations. Low $\mathrm{p} 53$ was seen by IHC in $62 \%$ of HGSOC associated with non-missense mutations. Most wild-type TP53 tumours $75 \%$, 6/8) displayed intermediate $p 53$ levels. The overall sensitivity of detecting a TP53 mutation based on classification as 'Low', 'Intermediate' or 'High' for $\mathrm{p} 53 \mathrm{IHC}$ was $99 \%$, with a specificity of $75 \%$. We suggest $\mathrm{p} 53 \mathrm{IHC}$ can be used as a surrogate marker of TP53 mutation in $\mathrm{HGSOC}$; however, this will result in misclassification of a proportion of TP53 wild-type and mutant tumours. Therapeutic targeting of mutp53 will require knowledge of both TP53 mutations and mutp53 expression.

TP53 is widely acknowledged as the most frequently mutated gene in human malignancy, with mutations identified in at least $50 \%$ of human cancers ${ }^{1-5}$. In the absence of cellular stress, wild-type p53 is maintained at low levels by the E3 ubiquitin ligase MDM2 that polyubiquitinates p53, marking it for proteasomal degradation. In response to stress, numerous mechanisms act to disrupt the MDM2-p53 association, resulting in stabilisation and activation of $\mathrm{p} 53^{6}$. Activated wild-type $\mathrm{p} 53$ promotes processes consistent with tumour suppression, including cell cycle inhibition, apoptosis, senescence, DNA repair and autophagy, as well as processes that oppose oncogenic metabolic reprogramming [reviewed in ${ }^{2}$. Mutant p53 (mutp53) results in loss of these tumour suppressive functions.

Human TP53 maps to chromosome 17p13.1 and is composed of 19,198 nucleotides spanning eleven exons, with the coding sequence commencing in exon 2 [reviewed in ${ }^{7}$ ]. Mutations in TP53 can occur throughout the coding sequence; however, around $80 \%$ are located in the DNA binding domain (DBD) encoded by amino acid residues 102 to 292 and encompassing six 'hot-spot' amino acids ${ }^{7-9}$. TP53 mutations include single base substitutions leading to missense or nonsense mutations, deletions or insertions leading to frameshifts, in-frame deletions or insertions, as well as mutations that affect splicing. Pathogenic mutations result in loss or abrogation of wild-type p53 activity, a major mechanism likely being the inability of mutp53 to bind to response elements in DNA, preventing its function as a transcription factor. Along with the loss of wild-type function, many TP53 mutations result in gain of an oncogenic function for the mutp53 protein. These 'gain-of-function' (GOF) TP53 mutations cause mutp53 to accumulate at high levels in cells, contributing to tumorigenesis and the development of drug resistance ${ }^{1}$. Not all p53 mutants that lead to accumulation of p53 are proven GOF mutations; however,

${ }^{1}$ Hormones and Cancer Group, Kolling Institute of Medical Research, Royal North Shore Hospital, University of Sydney, NSW 2065 Australia. ${ }^{2}$ Department of Anatomical Pathology, Royal North Shore Hospital and University of Sydney, NSW 2065 Australia. ${ }^{3}$ Hunter New England Local Health District, Royal North Shore Hospital, University of Sydney, Australia. ${ }^{4}$ Department of Obstetrics and Gynaecology, Royal North Shore Hospital, St Leonards, Australia. ${ }^{5}$ Mater Private and Royal North Shore Hospitals, Sydney, NSW, Australia. Correspondence and requests for materials should be addressed to D.J.M. (email: deborah.marsh@sydney.edu.au) 
certain mutants such as p.Arg175His, p.Gly245Ser, p.Arg248Trp, p.Arg248Gln and p.Arg273His have been shown to actively promote tumorigenesis [reviewed in ${ }^{2}$. Furthermore, a number of GOF mutations, including p.Arg175His and p.Arg273His have been linked to the development of chemoresistance ${ }^{10,11}$.

Given the broad role of p53 in malignancy, there is intense focus on its potential as a therapeutic target. Strategies include restoring normal transcriptional activity of mutp 53 by restoration of wild-type protein folding using drugs such as APR-246 that are currently recruiting for Phase Ib/II clinical trials for recurrent high-grade serous ovarian cancer (HGSOC) ${ }^{12}$ or zinc metallochaperones ${ }^{13}$. Alternatively, gene replacement therapy to reintroduce wild-type p53 into mutp53 cells, most often with an adenoviral vector (Adp53), has also been shown to suppress tumour growth ${ }^{14}$. It is clear that success of these different approaches will be influenced by the type of TP53 mutation present, and whether it results in accumulation of mutp53 in the cell, severe truncation of the p53 protein, or low to absent levels of $\mathrm{p} 53$

HGSOC is characterised by a high frequency of TP53 mutation. Worldwide, HGSOC is the eighth most frequent cause of cancer-related deaths in women ${ }^{15}$. More than $80 \%$ of women present with advanced disease (Stage III-IV), where 5 year survival is around $25 \%{ }^{16}$. The mainstay treatment is surgical debulking followed by combinations of platinum drugs and paclitaxel; however, the majority of women relapse within two years, ultimately develop broad chemoresistance and succumb to their disease ${ }^{17}$.

With the advent of massively parallel sequencing (MPS) and analyses of large data sets through The Cancer Genome Atlas, TP53 mutations have been identified at a frequency of approximately $96 \%$ in large HGSOC cohorts $^{18,19}$. It has recently been suggested that $100 \%$ of HGSOC are in fact TP53 mutant ${ }^{20}$. These figures are higher than first thought from reports that used less sensitive methods such as Sanger sequencing and/or only focussed on mutational 'hot-spot' regions of this gene ${ }^{21-24}$. TP53 is in fact the only gene that is frequently mutated at the somatic level in HGSOC, this malignancy otherwise being characterised by low prevalence (2-6\%) of recurrently mutated genes such as $B R C A 1, B R C A 2, R B 1$ and $N F 1$, and a long tail of infrequently mutated genes ${ }^{18}$. TP53 mutations are believed to occur early in tumorigenesis, likely in precursor lesions of ovarian cancer, highlighting the importance of mutp53 as a driver of this malignancy ${ }^{25-28}$.

Immunostaining for $\mathrm{p} 53$ has been used as a surrogate marker for the presence of a TP53 mutation in HGSOC, although precise validation of this practice by comparison to detailed sequencing data has been limited, especially in the context of MPS ${ }^{21,24,29}$. Here, we report the use of MPS to characterise TP53 mutations in a cohort of HGSOC in parallel with p53 immunohistochemical analyses. We note cut-offs based on our data related to percent positive $\mathrm{p} 53$ nuclei that should be used to increase the accuracy of p53 immunohistochemistry (IHC) as a surrogate marker of mutp53 and suggest that both p53 IHC and MPS should be considered to inform therapies targeting this protein. Further, we report TP53 mutations in previously uncharacterised cell line models of HGSOC.

\section{Results}

TP53 mutations identified using massively parallel sequencing in high- and low-grade serous ovarian cancer. Depth of coverage for data generated was approximately 850 reads per amplicon per sample. A mutation in TP53 was identified in 94\% (68/72) of HGSOC. Four HGSOC reported to be wild-type for TP53 underwent second review of archived tissue by an experienced pathologist [AJG] and remained classified as HGSOC. An additional four samples contained clear cell components and were excluded from this study (Fig. 1). The four low-grade serous ovarian cancer (LGSOC) samples contained wild-type TP53 (Table 1; Supplementary Table S1). The majority of mutations identified $(62 \%, 42 / 68)$ were missense, with all except one of these mutations located in the DBD of p53 (Fig. 2). The single missense mutation occurring outside of the DBD, p.Leu344Gln, was located in exon 10 of TP53. All missense mutations had been previously reported in The Cancer Genome Atlas ovarian study ${ }^{18}$ and/or the International Agency for Research on Cancer (IARC) TP53 database (Supplementary Table S1). TP53 mutations that had not been previously reported are indicated (Supplementary Table S1). Overall, $82 \%(56 / 68)$ of mutations occurred within the DBD. Frameshift, nonsense, in-frame insertion or splice mutations $(38 \%, 26 / 68)$ were identified in exons or flanking intronic sequence between exons 4-10.

Arginine 248 (Arg248) was the most frequently mutated amino acid, with approximately 9\% (6/68) of mutations (p.Arg248Gln and p.Arg248Trp) occurring at this codon, followed by Arg175 with approximately 6\% (4/68) of mutations (p.Agr175His) and Arg342 with approximately 4\% (3/68) of mutations (p.Arg342* and p.Arg342 Glufs*2) occurring at this amino acid (Fig. 2). The frequency of mutation type we observed in our cohort was not dissimilar to that reported in recent studies ${ }^{18,19}$. The presence or location of a TP53 mutation did not influence overall patient survival (Supplementary Fig. S1).

Validation of TP53 by Sanger sequencing in HGSOC. Of the 68 TP53 mutations identified by MPS, Sanger sequencing of the identical DNA sample analysed in a blinded manner was able to identify 61 ( $90 \%)$. Of the seven samples that were incorrectly determined to be TP53 wild-type by Sanger sequencing, re-analysis with knowledge of the mutation that was identified by MPS identified small variant peaks in five samples consistent with presence of the identified mutation (Fig. 3). In the remaining two samples, no variant was detected by Sanger sequencing alone (Fig. 3). This data therefore suggests that allele frequencies of less than approximately $25 \%$ are unable to be reliably detected by Sanger sequencing.

Correlation between TP53 mutation status and p53 immunohistochemistry in HGSOC and LGSOC. The overall sensitivity of detecting a TP53 mutation based on classification of immunohistochemical staining as 'Low' or 'High' was 99\% (95\% Confidence Interval [CI] 91-99\%), with a specificity of 75\% (95\% CI 36-96\%). For tumours graded 'High' (68\%; 52/76), all except one was TP53 mutant (98\%; 51/52). For tumours graded 'Low' $(24 \% ; 18 / 76)$, all except one were TP53 mutant $(94 \% ; 17 / 18)$. The remaining tumours were scored 'Intermediate' (9\%; 7/76), of which six were TP53 wild-type (86\%; 6/7) (Fig. 4a). The actual number of nuclei staining positive for p53 in the 'Intermediate' category ranged from 8 to 65\% (Supplementary Table S2). 


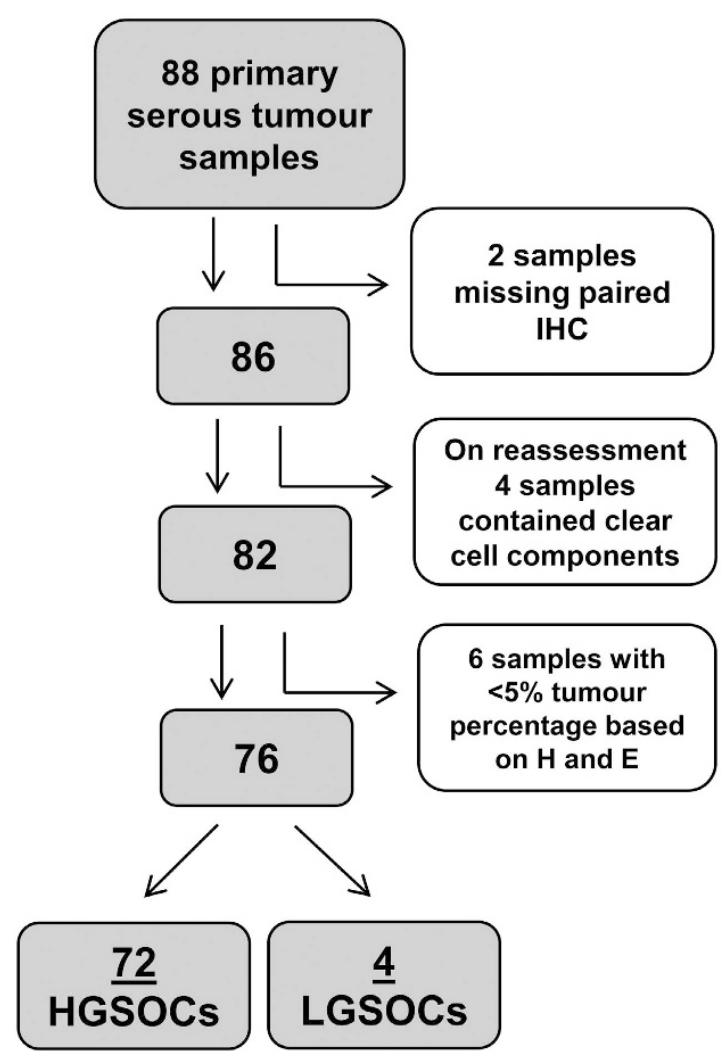

Figure 1. Flow diagram summarising inclusion and exclusion criteria from the primary tumour cohort. Eighty-eight primary ovarian tumours underwent MPS for TP53. Two samples were excluded as paired p53 IHC was unable to be obtained. Upon pathological reassessment, 4 samples were excluded due to the identification of clear cell components. A further 6 samples were excluded as they contained $<5 \%$ tumour cells based on hematoxylin and eosin staining. Of the 76 remaining samples, 72 were HGSOC and 4 were LGSOC.

\begin{tabular}{|l|c|c|c|c|}
\hline & Frequency & High IHC & Low IHC & Intermediate IHC \\
\hline \multicolumn{5}{|l|}{ High-grade serous ovarian cancer } \\
\hline Frameshift & 9 & $2(22 \%)$ & $7(78 \%)$ & $0(0 \%)$ \\
\hline Nonsense & 11 & $4(36 \%)$ & $6(55 \%)$ & $1(9 \%)$ \\
\hline Splice & 5 & $2(40 \%)$ & $3(60 \%)$ & $0(0 \%)$ \\
\hline Missense & 42 & $42(100 \%)$ & $0(0 \%)$ & $0(0 \%)$ \\
\hline In-frame insertion & 1 & $1(100 \%)$ & $0(0 \%)$ & $0(0 \%)$ \\
\hline Wild-type & 4 & $1(25 \%)$ & $1(25 \%)$ & $2(50 \%)$ \\
\hline Total Mutations & $68(94 \%)$ & & & \\
\hline Total Samples & $\mathbf{7 2}$ & & & \\
\hline Low-grade serous ovarian cancer & 4 & $0(0 \%)$ & $0(0 \%)$ & $4(100 \%)$ \\
\hline Wild-type & 0 & & & \\
\hline Total Mutations & $\mathbf{4}$ & & & \\
\hline Total Samples & 4 &
\end{tabular}

Table 1. TP53 mutations and immunohistochemical classification for high- and low-grade serous ovarian cancer. Tumours with $\geq 70 \%$ positive p53 nuclei were categorized as 'High' IHC. Tumours with $>5 \%$ and $<70 \%$ positive p 53 nuclei were categorized as 'Intermediate' IHC, while samples with $\leq 5 \%$ positive p53 nuclei were categorized as 'Low' IHC.

Representative images of these staining patterns are shown in Fig. $4 \mathrm{~b}$ and Supplementary Figs S2 and S3. The DO-7 antibody used was suitable for detecting all variants identified in our study by MPS.

All missense mutations were scored as 'High' for expression of the $\mathrm{p} 53$ protein by IHC, indicating that these mutations were associated with increased levels of mutp53. Considerable variability was observed in p53 staining levels in samples containing stop, frameshift, in-frame insertion or splice mutations $(\mathrm{N}=26)$ (Fig. 4a). While the majority of samples in each non-missense mutation category were scored as 'Low' for p53 IHC (7 of 9 frameshift mutations, 6 of 11 nonsense mutations and 3 of 5 splice mutations), a single nonsense mutation, p.E298* (exon 8), 


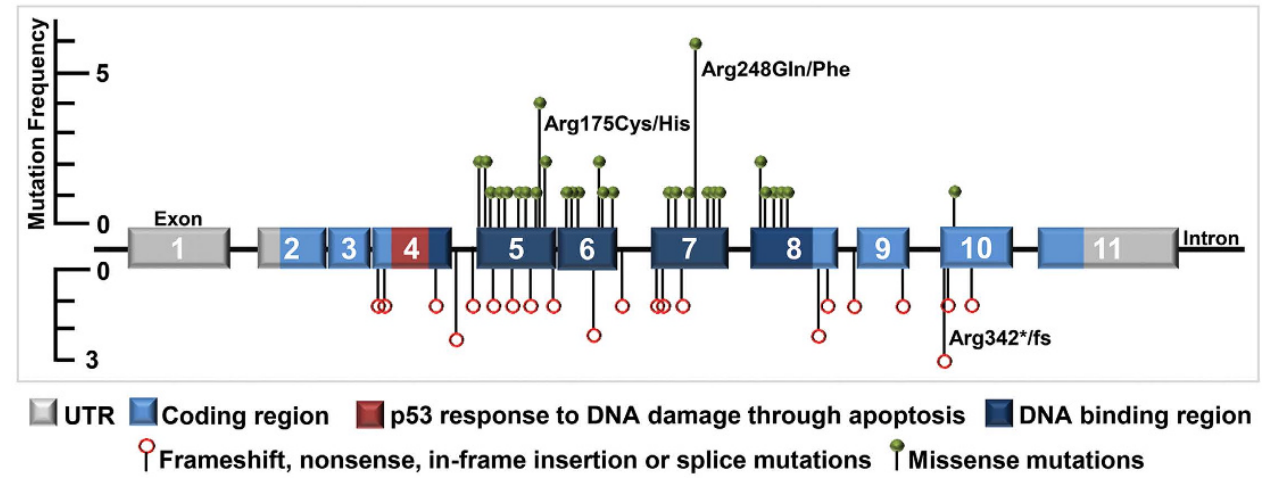

Figure 2. Distribution of TP53 mutations. Grey exons mark the un-translated regions (UTR), light blue exons denote the coding regions, while dark blue exons within the coding region correlate with the DNA binding domain. The dark red region marks the p53 response to DNA damage through apoptosis area. Intronic areas are shown by the black line joining exons. All 68 mutations identified in this study are displayed as lollypop symbols, with missense mutations above the gene marked as green circles, while all 'other' mutation types (nonsense, frameshift, in-frame insertion and splice site) are displayed below the gene as open red circles. Mutations occurring more than once are indicated.

was classified as 'Intermediate'. The nine remaining mutations were classified as 'High' for p53 IHC, consisting of 2 of 9 frameshift mutations (p.Phe328fs in exon 9 and p.Arg342fs in exon 10); 4 of 11 nonsense mutations, all in exon 10 (two independent samples with p.Arg342*, p.Glu343* and p.Glu349*); 2 of 5 splice site mutations (c.376-2A > G and c.920-1G > A) (Fig. 4a); and a single in-frame insertion duplication in exon 7, c.723_724dupACCATCCACTACAACTACATGTGTAACAGTTCC. The locations of all twenty-six non-missense mutations are summarised along with their p53 IHC pattern (Fig. 5).

Twelve (12/16, 75\%) mutations associated with 'Low' expression were located within the DBD compared with two $(2 / 9,22 \%)$ of the mutants associated with 'High' expression. The mutation associated with 'Intermediate' expression was not located in the DBD. Furthermore, all of the mutations associated with 'Low' expression occurred before exon 9 compared with just $22 \%$ of the mutations associated with 'High' expression. All LGSOC $(\mathrm{N}=4)$ were wild-type for TP53, and were graded 'Intermediate' for p53 IHC. Wild-type TP53 HGSOC $(\mathrm{N}=4)$ consisted of one sample graded as 'High' for p53 IHC, two 'Intermediate' and another as 'Low' (Fig. 4a). The percentage of tumour cell nuclei positive for p53 immunostaining for each mutation category, including wild-type, is depicted in Fig. 6. Whether p53 staining patterns were designated 'Low', 'Intermediate' or 'High' did not affect overall patient survival (Supplementary Fig. S1).

TP53 mutations and p53 protein expression in previously uncharacterized HGSOC and clear cell ovarian cancer cell lines. Among the cell lines examined, Western immunoblotting showed the greatest basal p53 expression in OV202, followed by OV207. OV167 expressed a lower level of basal p53 compared to the wild-type p53 A2780 cells (Fig. 7a). Only OV202 displayed a statistically significant difference in p-p53 abundance from the other cell lines ( $p=0.000005-0.000016$; Fig. $7 \mathrm{~b})$. At the transcript level, all cell lines showed a significant difference between TP53 transcripts, OV167 $<$ A2780 < OV202 $<$ OV207 $(p=0.000002-0.046$; Fig. 7c). OV202 and OV207 were found to have the TP53 missense mutations c.722C > T (p.Ser241Phe) and c.818G > A (p.Arg273His) respectively. OV167 had the nonsense mutation c.892G > T (p.Glu298*) (Fig. 7d). Further, immunostaining of a cell pellet of OV167 showed an "Intermediate" staining pattern similar to the tumour sample \#353-09 shown to have the identical mutation (Supplementary Fig. S4). The mutant allele frequency for each of these cell lines was $\geq 99 \%$ as determined by MPS and our analysis pipeline.

\section{Discussion}

Mutation of TP53 is pervasive in HGSOC; however, whether these mutations lead to accumulation of mutp53 and possible dominant-negative and/or gain-of-function effects, or low to absent levels of mutp53 is dependent upon individual mutations. Using MPS, we identified 94\% (68/72) of HGSOC with TP53 mutation and 100\% (4/4) of LGSOC were wild-type for TP53, similar to recent studies ${ }^{18,19,24}$. Pathological reassessment of the four TP53 wild-type HGSOC did not change the initial diagnosis of HGSOC. When correlated with p53 IHC data for the same specimens, we showed an overall sensitivity of $99 \%$ for detecting a TP53 mutation based on an IHC score of $\leq 5 \%$ or $\geq 70 \%$ percent $\mathrm{p} 53$ positive nuclei; however, this test showed a lower specificity of $75 \%$. In a cohort of HGSOC where the expectation is that the vast majority of samples will be TP53 mutant, p53 IHC may be considered an acceptable surrogate marker for TP53 mutation. In tumour types where the frequency of somatic TP53 mutation is expected to be much lower, the lower specificity of this test may impact on its value as a surrogate marker of TP53 mutation.

There is general agreement in the literature that both high and low, or entirely absent, p53 expression is correlated with mutant p53, while expression levels between these two extremes are thought to represent wild-type p53. In studies of HGSOC, high levels of p53 positive nuclei indicative of mutp53 have been described as $>30 \%{ }^{30}$, $>50 \%{ }^{31,32}$ and $\geq 60 \%^{21}$, although it is noted in one report that the majority of high expressors showed $>85 \% \mathrm{p} 53$ positive nuclei ${ }^{31}$. In the current study, the cut-off for high p 53 expression was $\geq 70 \%$. Reduction of this cut-off 


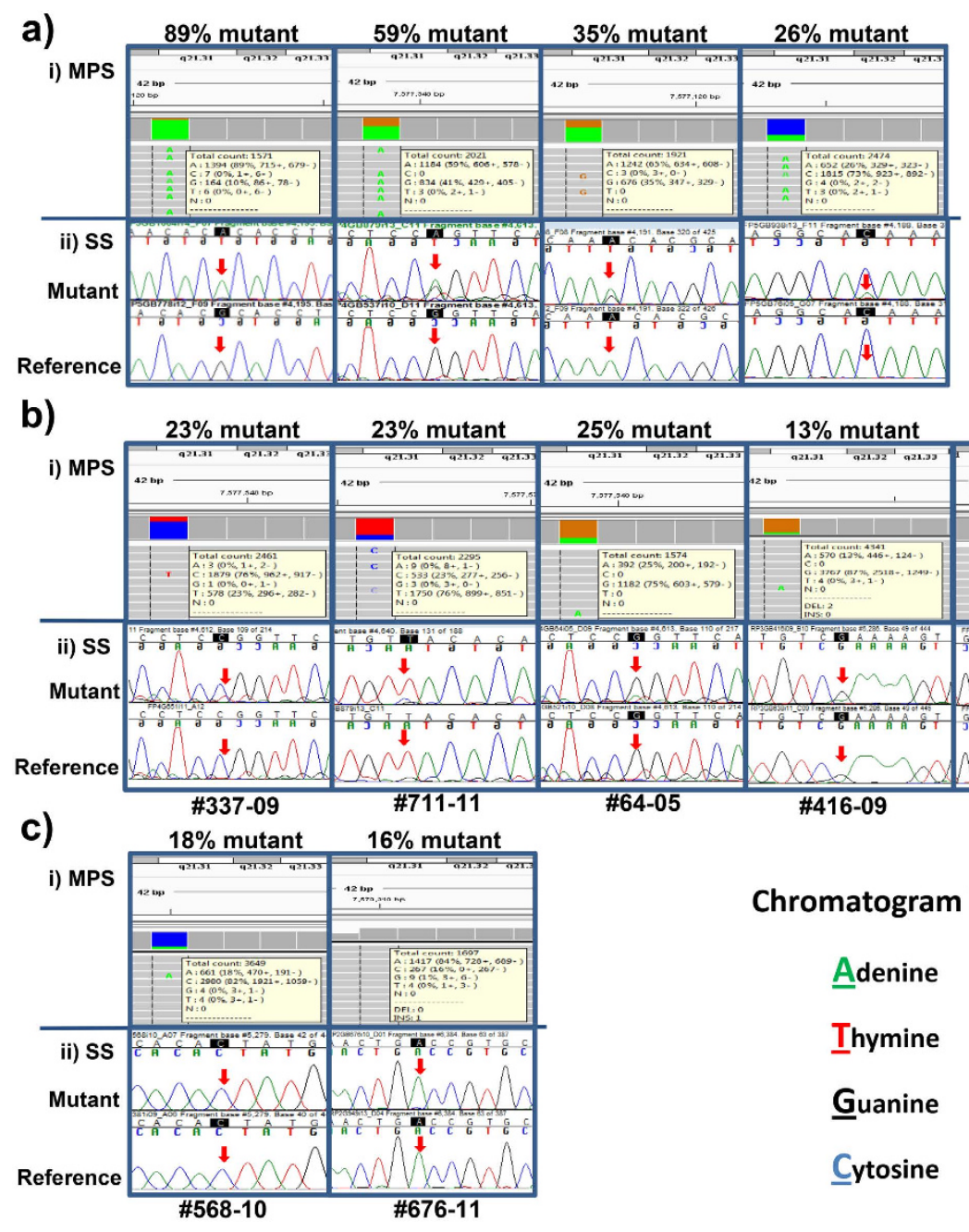

Figure 3. Comparison of the ability of next generation and Sanger sequencing to detect TP53 mutations. Top panels (a-c) display MPS data viewed using IGV software (i). Lower panels display Sanger sequencing (SS) chromatograms of mutant and wild-type reference samples viewed using Sequencher software (ii). Red arrows denote presence of mutations in SS, initially discovered by MPS. (a) Shows four mutations with frequencies of the mutant allele between $26-89 \%$ determined by MPS that were able to be detected by SS. (b) Shows five mutations with frequencies of the mutant allele between $13-29 \%$, which were initially not detected by SS; however, with the knowledge of MPS results, were identified in the chromatograms. (c) Shows two samples with frequencies of the mutant allele between 16-18\% determined by MPS that were unable to be detected at all in SS chromatograms.

to lower levels previously published would have resulted in misclassification of several wild-type TP53 tumours as mutant and lowered the overall specificity of this test. A lower cut-off for high expression would; however, have correctly classified tumour \#353-09 that displayed 55\% p53 positive nuclei and was misclassified under our criteria.

Low levels of p53 expression correlated with the presence of a mutation have been described as completely negative ${ }^{21,31}$, although tumours with wild-type TP53 have also been reported to have low p53 levels, $\leq 10 \%$ positive nuclei ${ }^{21}$, potentially complicating clear discrimination between mutant and wild-type p53. In the current study, 10 tumours showed complete absence of p53, one of which was wild-type. A further 7 tumours, all TP53 mutant, showed $<5 \%$ positive $\mathrm{p} 53$ nuclei $(1-4 \%)$. We suggest that even with careful optimisation of p53 immunohistochemistry, this lower range of p53 expression will pose problematical for the discrimination of all wild-type from mutp53 tumours.

The majority of TP53 mutations identified in this study $(82 \% ; 56 / 68)$ were located in the p53 DBD. Hot-spot codons for mutation included p.Arg248 and p.Arg175 residing within the p53 DBD, that have previously been recognized as TP53 mutational hot-spots in human malignancy ${ }^{2}$, as well as p.Arg342. No differences in overall survival in HGSOC were observed that could be correlated with presence or absence of a TP53 mutation, mutation type or location; however, we could not exclude the possibility that specific mutations, or mutations at certain amino acids, may in fact influence patient survival. 


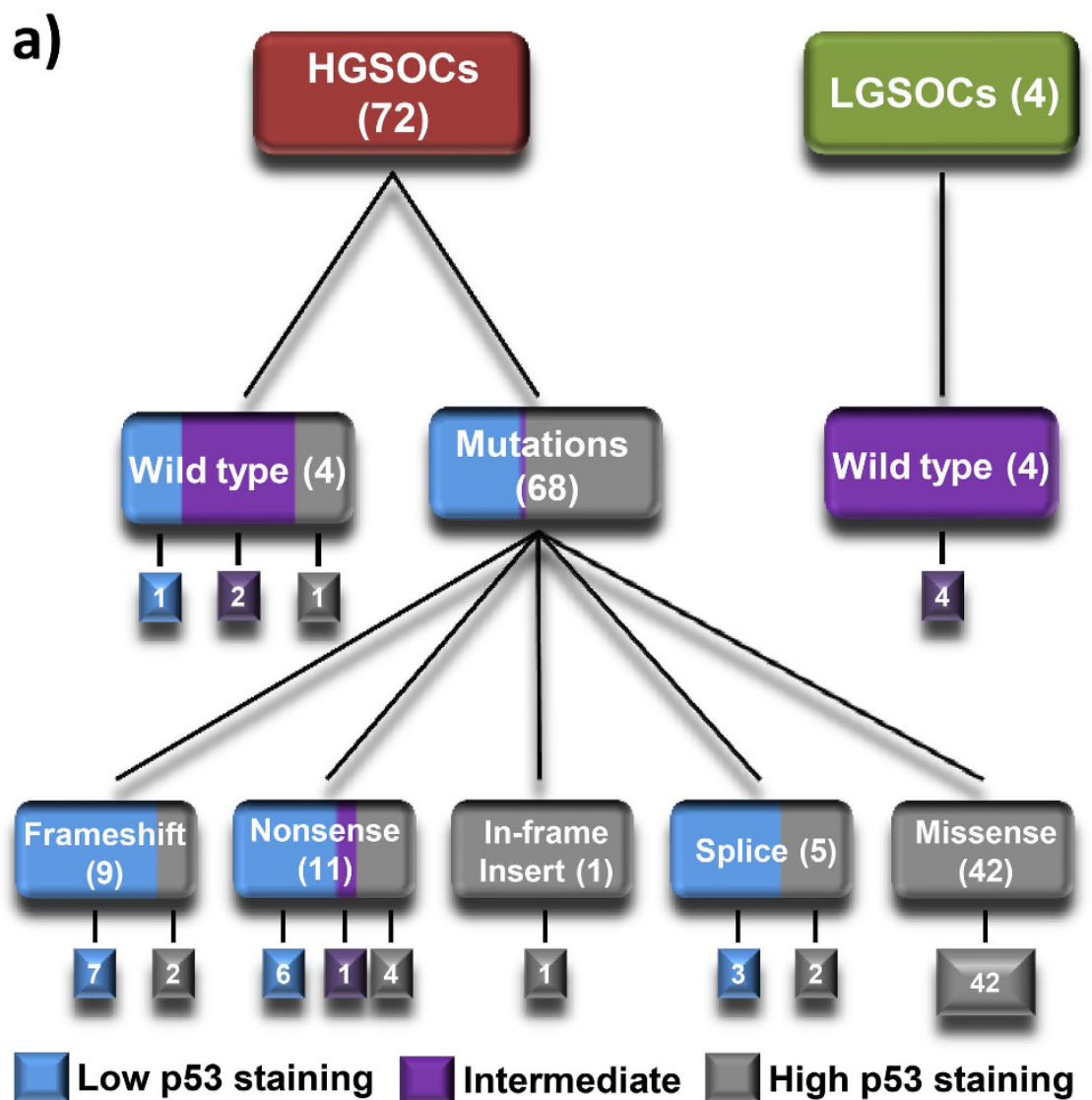

\section{b)}
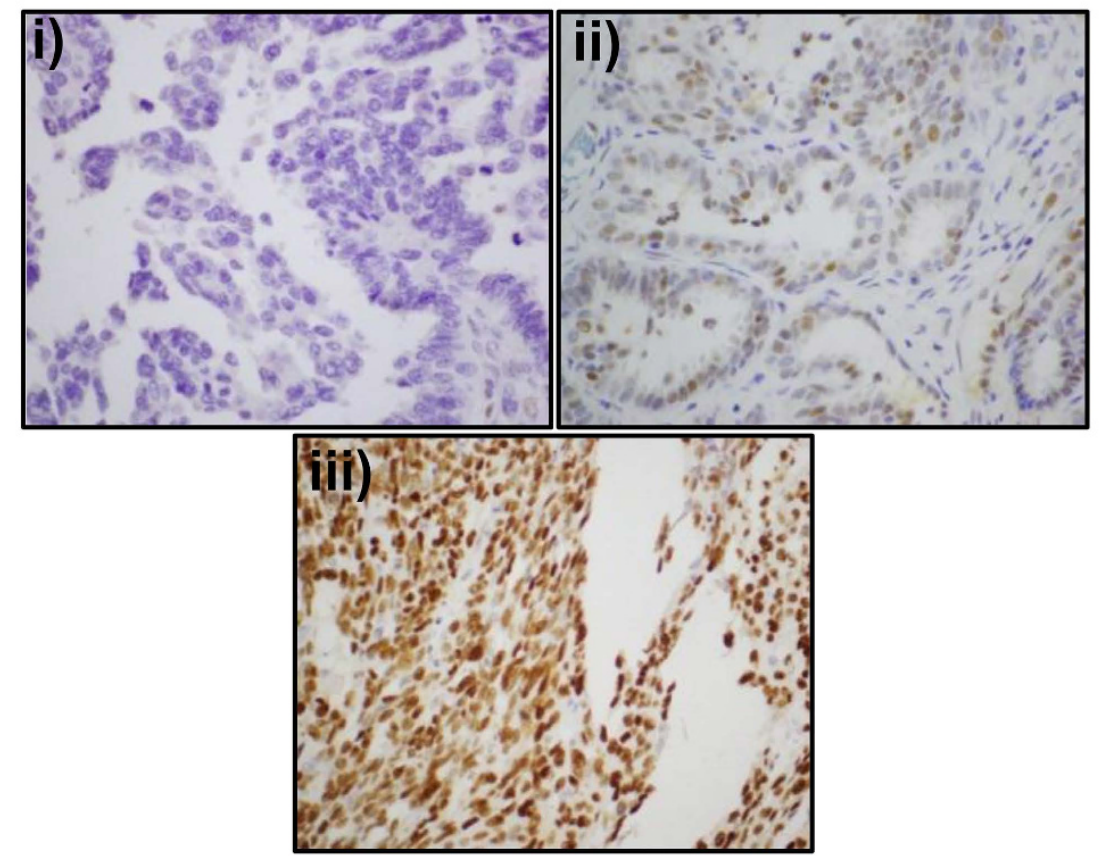

Figure 4. p53 immunohistochemistry and TP53 status in HGSOC and LGSOC. (a) Immunohistochemistry scores ('Low', 'Intermediate' or 'High') correlated with TP53 mutation status. (b) Representative images of p53 immunohistochemistry showing (i) 'Low', (ii) 'Intermediate' and (iii) 'High', scores. 


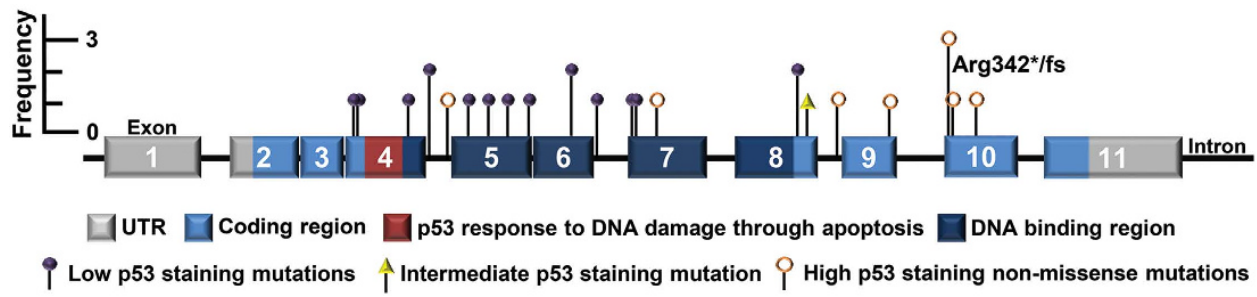

Figure 5. Distribution of non-missense low and high expression TP53 mutations. Grey exons mark the untranslated regions (UTR), light blue exons denote the coding regions, while dark blue exons within the coding region correlate with the DNA binding domain. The dark red region marks the p53 response to DNA damage through apoptosis area. Intronic areas are shown by the black line joining exons. All 26 non-missense mutations identified in our cohort are displayed along TP53. Mutations that resulted in accumulated p53 are identified with open orange circles. The mutation that resulted in 'Intermediate' p53 staining is identified as a yellow triangle. Mutations that resulted in loss of p53 expression are identified as closed purple circles. Mutations occurring more than once are indicated.

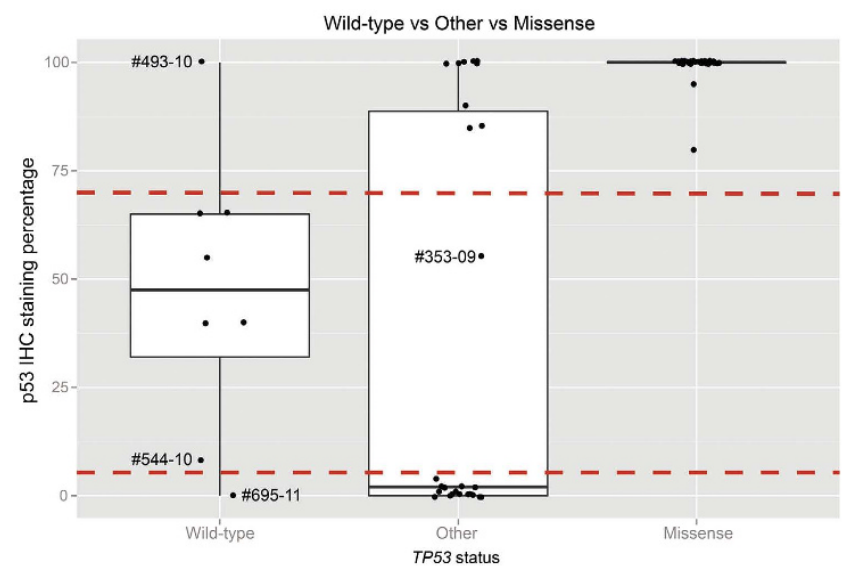

Figure 6. Correlation of wild-type and mutant p53 levels with TP53 mutation status (wild-type, missense and all 'other' mutations). While all missense TP53 mutants resulted in $\geq 70 \%$ tumour cell nuclei staining positive for p53, there was considerable variation amongst p53 staining patterns for wild-type (including both HGSOC and LGSOC) and non-missense TP53 mutations. While the majority of wild-type TP53 samples clustered between $8-65 \%$ tumour cell nuclei staining positive for $\mathrm{p} 53$ (denoted by the broken red line), one HGSOC sample demonstrated Low staining (\#695-11; 0\%) and one High (\#493-10; 100\%). Furthermore, there were two distinct clusters of non-missense mutations, with tumour cell nuclei staining positive for p53 between $80-100 \%$ and $0-4 \%$, that could not further be discriminated by whether the mutations were nonsense, splice site, an in-frame insertion or frameshifts. Furthermore, a single nonsense mutation displayed $55 \%$ of tumour cell nuclei staining positive for p53 (\#353-09).

analyses were performed in a blinded fashion. The lower sensitivity of this technique in comparison to MPS may at least partly explain the lower frequency of TP53 mutations in HGSOC reported in earlier studies ${ }^{21,23}$. This will have impacted on the accuracy of previous studies where attempts have been made to correlate TP53 mutation and $\mathrm{p} 53 \mathrm{IHC}^{21}$. In the current study, Sanger sequencing was unable to robustly identify mutations where the frequency of the mutated allele determined by MPS was less than $25 \%$. This limit of detection is supported by other studies $^{33,34}$.

The majority (62\%) of mutations identified in HGSOC were missense, all occurring in the p53 DBD with the exception of p.Leu344Gln in exon 10. All tumours with missense mutations were scored as 'High' by p53 IHC, consistent with accumulation of mutp53. This data suggests that p53 IHC is a strong surrogate marker of TP53 missense mutation; however, it was not specific for this class of mutation. Four mutations in exon 10 classified as nonsense (two tumours with p.Arg342*, p.Glu343* and p.Glu349*) and two with frameshifts (p.Phe328Hisfs*7 in exon 9 and p.Arg342Glufs*2 in exon 10) also resulted in accumulation of mutp53 and a score of 'High' for IHC. All of the nonsense and frameshift mutations associated with high levels of mutp53 would be predicted to truncate p53 towards its carboxyl-terminus. Splice site mutations (c.376-2A $>$ G in intron 4 and c.920-1G $>$ A in intron 8), as well as an in-frame insertion of a duplication in exon 7, also resulted in accumulation of mutp53 and a 'High' p53 IHC score. The remaining nonsense, frameshift and splice site mutations resulted in 'Low' p53 IHC scores, with the exception of a single nonsense mutation, p.Glu348* in exon 8, that demonstrated an 'Intermediate' p53 staining pattern. Two distinct clusters of non-missense mutations in the context of cellular 
a

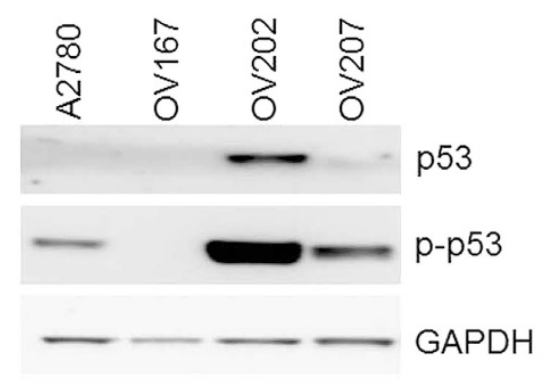

C

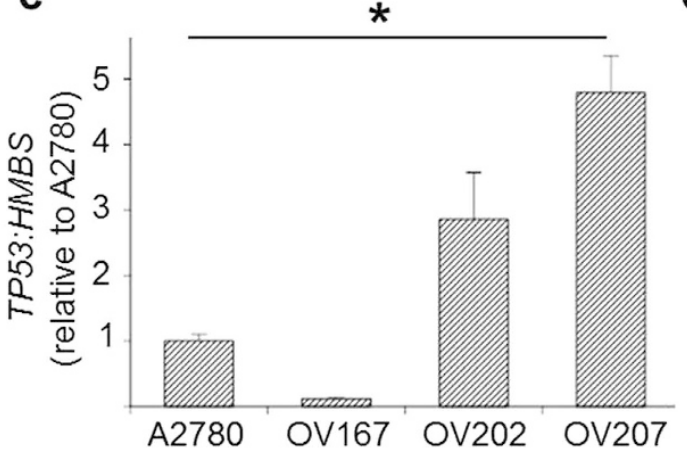

b

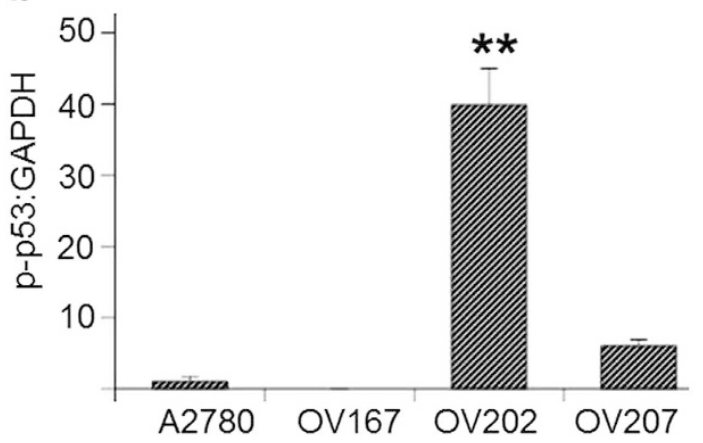

d Cell Line Exon TP53 Mutation OV167 OV202 8 c.892G>T p.Glu298* 7 c.722C $>$ T p.Ser241Phe OV207

Figure 7. p53 protein and transcript levels in previously uncharacterized HGSOC cell lines (OV167 and OV202) and clear cell carcinoma cell line (OV207) compared to a wild-type p53 cell line (A2780). (a) Representative Western blots of basal levels of p53 and phosphorylated p53 (Ser15) (p-p53). (b) Graphical representation of protein levels of basal p-p53 in TP53 mutant ovarian cancer cell lines relative to A2780 (N=3, mean \pm S.E.M, ${ }^{* *} P<0.01$ ). (c) Graphical representation of TP53 transcript levels relative to the reference gene HMBS in ovarian cancer cell lines compared to A2780 $\left(\mathrm{N}=3\right.$, mean \pm S.E.M, $\left.{ }^{\star} P<0.05\right)$. (d) TP53 mutations identified by our Fluidigm-MiSeq analysis pipeline in previously uncharacterised ovarian cancer cell lines.

accumulation of mutp53 were present in this study; however, they could not be clearly defined based on mutation type or location.

Four HGSOC were identified as wild-type for TP53. These fresh frozen specimens contained between $30-80 \%$ tumour cells. In the absence of mutation or stimulus as the result of DNA damage, wild-type p53 would be expected to be present at low levels in the cell ${ }^{6}$. Of the HGSOCs that were wild-type for TP53, one showed 'High' p53 IHC, two 'Intermediate' and one 'Low'. As expected, the four LGSOCs were TP53 wild-type, and all showed 'Intermediate' staining for p53 IHC. It is possible that the wild-type TP53 tumours demonstrating 'High' or 'Low' levels of accumulated p53 may have other abnormalities, such as amplification of MDM2 or MDM4 that has previously been associated with some, but not all, TP53 wild-type HGSOC ${ }^{29}$. The literature remains conflicted as to whether differing levels of mutp53 are associated with survival24,29,31,32,35-37; however, we did not find any association between overall survival and levels of mutp53 expression in this study (Supplementary Fig. S1).

Recent genomic assessment of cell line models of HGSOC has identified cell lines believed to be stronger models for studying this cancer based on their genomic similarity to primary tumours ${ }^{38,39}$. All cell lines ranking as strong models for HGSOC were TP53 mutant. Two HGSOC cell lines not included in these published studies, OV167 and OV202, contained a nonsense and missense TP53 mutation respectively. A third cell line established from a clear cell adenocarcinoma, OV207, also contained a missense TP53 mutation. While our reporting of TP53 mutation status in these cell lines reflects only part of the extensive genomic analyses of cell lines performed by other studies ${ }^{38,39}$, it does support the likelihood that these are suitable cell line models for studying this malignancy.

In summary, we suggest that p53 IHC may be used with care as a surrogate marker of TP53 mutation by designating specimens demonstrating 'Intermediate' p53 staining as most likely p53 wild-type. However, detailed knowledge of mutation effect determined by the combination of MPS, mutation location in TP53 and levels of p53 expression may be particularly important when considering options for therapeutic targeting of mutant and wild-type p53 that are currently in development ${ }^{40}$. The success of therapeutic targeting of mutant p53 will in large part depend on knowledge of both the level of p53 expression and the exact nature of the mutation. While this study has focussed on HGSOC, combining MPS of TP53 with p53 IHC should also be considered when investigating different therapeutic strategies for targeting p53 in other human malignancies. Sensitivities and specificities of these tests should be determined for other malignancies that demonstrate a range of TP53 mutation frequencies. 


\begin{abstract}
Materials and Methods
Tumour Samples. Our cohort consisted of 88 gynecological tumours (serous ovarian, fallopian tube and peritoneal carcinomas) collected from 2004 to 2014 from three hospitals (Royal North Shore Hospital, North Shore Private and The Mater Hospital-North Sydney, Sydney, Australia). Seventy-two HGSOC and four low grade serous tumours (LGSOC) were included in the final analysis. Rationale for specimen exclusion is described in Fig. 1. Eighty-two percent (59/72) of HGSOCs were at an advanced stage, classified as Stage III or IV. Survival and other clinical data were available for the majority of patients (Supplementary Table S3). Survival times were defined as time from date of surgery to last follow up or death as recorded in hospital medical records, doctors' rooms and publicly available death notices. All snap frozen tumour samples were collected and stored in the Kolling Institute of Medical Research (KIMR) Gynecological Tumour Bank. Matched formalin fixed paraffin embedded samples were obtained from one of two diagnostic pathology laboratories (Pacific Laboratory Medicine Services, PaLMS or Douglass Hanly Moir Pathology, Sydney, Australia). Ethical approval for this project was obtained from the Northern Sydney Local Health District Human Research Ethics Committee (Protocol: 108-243 M). Written informed consent was obtained from all subjects. All methods were carried out in accordance with the approved guidelines. All tumour tissue was reviewed by a pathologist [AJG] to confirm diagnosis, histological grade and pathological stage. Sequential sections were ascertained from frozen tumours for determination of percent tumour cells following hematoxylin and eosin staining, and for DNA extraction (Supplementary Table S1). Tumours containing at least $5 \%$ of tumour cells were selected for this study.

DNA was prepared from approximately $30 \mathrm{mg}$ fresh frozen tumour tissue, homogenised in phosphate buffered saline using a Retsch MM 301 Mixer Mill (MEP Instruments Pty. Ltd., NSW, Australia). Tissue was shaken three times for 90 seconds at the highest frequency until liquefied. Protein was digested overnight at $56^{\circ} \mathrm{C}$ with proteinase K (Qiagen Pty Ltd, Chadstone, VIC, Australia). DNA was extracted using the DNeasy Blood and Tissue Kit and QIAcube (Qiagen Pty Ltd, Chadstone, VIC, Australia). DNA concentration was determined by Qubit Fluorometric Quantitation (Life Technologies Australia Pty. Ltd., Mulgrave, VIC, Australia) and 260:280 and 260:230 ratios determined using a NanoDrop ND-1000 spectrophotometer (Thermo Fisher Scientific Australia, Scoresby, VIC, Australia).
\end{abstract}

Ovarian Cancer Cell Lines. Three ovarian cancer cell lines that had not been previously characterised for TP53 status, HGSOC cell lines OV167 and OV202, and the clear cell adenocarcinoma line OV207, were analysed (cell lines were kindly provided by Drs C. Conover and K. Kalli, Mayo Center, Rochester, MN, USA) ${ }^{41}$. All cell lines were cultured in RPMI 1640 (Gibco, Life Technologies Australia Pty Ltd, Mulgrave, VIC, Australia) supplemented with 10\% (volume/volume) fetal bovine serum (SAFC Bioscience, Brooklyn, VIC, Australia) at $37^{\circ} \mathrm{C}$ with $5 \% \mathrm{CO}_{2}$ as previously described ${ }^{42}$. Cells were grown to $80 \%$ confluence prior to harvesting for DNA extraction. DNA was extracted using the DNeasy Blood and Tissue Kit and QIAcube (Qiagen Pty Ltd, Mulgrave, VIC, Australia).

Cell lines were authenticated by short tandem repeat profiling using the AMPFLSTR ${ }^{\circledR}$ Identifiler $^{\circledR}$ PCR Amplification Kit (Applied Biosystems), a 16 loci (15 STR loci plus Amelogenin) STR multiplex kit performed by CellBank Australia (Westmead, New South Wales, Australia). Authentication profiles are available for OV202 and OV167 $7^{42}$ and OV207 (Supplementary Table S4).

Approximately $3 \times 10^{6}$ of the TP53 missense HGSOC cell line OVCAR-3, p53 null cell line SK-OV-3, TP53 wild-type line A2780 and TP53 nonsense mutation line OV167 were harvested, washed twice with PBS and centrifuged at $400 \mathrm{RCF}$ for 3 minutes. Supernatant was discarded, cells resuspended in $100 \mu \mathrm{l}$ of $1 \%$ agarose (made up in PBS) and left at room temperature for 5 minutes for the agarose to set. Cell plugs were fixed using $10 \%$ formalin for $6 \mathrm{~h}$ before being dehydrated by changes in graded alcohol and xylene and embedded in a paraffin block. Cell lines underwent immunohistochemistry for p53 as described.

Western Immunoblotting and Gene Expression. Cells were lysed in RIPA buffer (Sigma-Aldrich, Castle Hill, NSW, Australia) containing Protease Inhibitor Cocktail (Sigma-Aldrich), phenylmethylsulfonyl fluoride (PMSF; Sigma-Aldrich) and the phosphate inhibitor cocktail PhosStop (Roche Diagnostics, Castle Hill, NSW, Australia). Thirty $\mu \mathrm{g}$ of total protein was added to $4 \times$ NuPAGE $^{\circledR}$ LDS Sample Buffer (Thermo Fisher Scientific, Waltham, MA, USA), followed by heating at $70^{\circ} \mathrm{C}$ for 10 minutes. Cell lysates were electrophoresed on $4-12 \%$ Bis-Tris gels (Life Technologies, Thornton, NSW, Australia) at $180 \mathrm{~V}$ for one hour followed by transfer to a nitrocellulose membrane (Amersham ${ }^{\mathrm{TM}}$ Protran $^{\circledR}$ Supported Western Blotting Membrane, GE Healthcare, Sigma-Aldrich) using a wet transfer system at $100 \mathrm{~V}$ for 1.5 hours (Bio-Rad Laboratories, Gladesville, NSW, Australia). Membranes were blocked with 5\% skim milk for one hour at ambient temperature, and probed overnight at $4{ }^{\circ} \mathrm{C}$ with primary antibody (p53 [1C12] mouse mAb, Phospho-p53(Ser15) rabbit polyclonal antibody, or GAPDH [14C10] rabbit mAb; all from Cell Signaling Technology, Danvers, MA, USA). Membranes were probed with peroxidase labeled secondary antibodies (anti-rabbit IgG HRP-linked whole antibody or anti-mouse IgG-HRP, both from GE Healthcare, VWR International, Murarrie, Queensland, Australia) for one hour. Chemiluminescent signal was detected by SuperSignal ECL Dura reagent (Pierce, Rockford, IL, USA) and visualised using the Fujifilm LAS-4000 imaging system (Berthold Australia Pty. Ltd., Bundoora, VIC, Australia). Quantitation was undertaken using Multi Gauge 3.0 (Fujifilm Australia Pty. Ltd., Brookvale, NSW, Australia).

Total RNA was extracted from all cell lines using the miRNeasy Mini Kit (Qiagen, Doncaster, VIC, Australia) and automated using a QIAcube (Qiagen). $500 \mu \mathrm{g}$ RNA was reverse transcribed using Superscript III reverse transcriptase (Life Technologies). Quantitative real-time RT-PCR was performed in triplicate using a TaqMan Gene Expression Assay for p53 (Hs01034249_m1; Life Technologies) and an endogenous reference gene (hydroxymethylbilane synthase (HMBS); Hs00609297_m1, Life Technologies), using iTaq Universal Probes Supermix (Bio-Rad Laboratories) on a 7900HT Fast Real-Time PCR System (Applied Biosystems). Reagents were aliquoted using an epMotion 5070 robot (Eppendorf South Pacific Pty Ltd., North Ryde, NSW, Australia). 
p53 Immunohistochemistry. Immunohistochemistry for p53 was performed on formalin fixed paraffin embedded tissue sections using a commercially available mouse monoclonal anti-human antibody (Protein Clone DO-7, cat. \#M7001, Dako, CA, USA) at a dilution of 1 in 50. Staining was performed on a representative whole section using an automated staining platform - the Leica BOND-III ${ }^{\mathrm{TM}}$ autostainer (Leica Biosystems, Mount Waverley, Victoria, Australia). Heat Induced Epitope Retrieval (HIER) was performed for 30 minutes at $97^{\circ} \mathrm{C}$ in the manufacturer's acidic retrieval solution (ER1: VBS part no: AR9961). Stained slides were examined by an experienced surgical pathologist [AJG] who was blinded to molecular data. The percentage of cells showing positive nuclear staining was estimated and reported in three categories: $\geq 70 \%$ positively stained nuclei (High); $>5 \%$ and $<70 \%$ stained nuclei (Intermediate); $\leq 5 \%$ positively stained nuclei (Low).

TP53 genomic PCR with the Fluidigm Access ArrayTM System and Massively Parallel Sequencing (MPS). DNA from tumour and cell line samples were processed for MPS using the Access Array ${ }^{\mathrm{TM}}$ BRCA1/ BRCA2/TP53 Target-Specific Panel (Fluidigm, South San Francisco, CA, USA). The 48.48 Access Array ${ }^{\mathrm{TM}}$ integrated fluidic circuits (IFC) was used, including target specific primers containing a common sequence tag (CS1 or CS2) and Illumina adaptors PE1 and PE2. A sample specific barcode was located on the reverse sequence (PE1_CS1 Forward Primer, 5'-AATGATACGGCGACCACCGAGATCTACACTGACGACATGGTTCTACA-3', 47 bp; PE2_BC_CS2 Reverse primer, 5'-CAAGCAGAAGACGGCATACGAGAT[sample specific barcode] TACGGTAGCAGAGACTTGGTCT-3', $56 \mathrm{bp}$ ). This array enabled 92\% coverage of TP53 using 16 primer pairs generating amplicons of between 191-209 base pairs. Five $\mu \mathrm{l}$ of DNA $(50 \mathrm{ng} / \mu \mathrm{l})$ was added to the array and run on the Fluidigm Biomark $\mathrm{HD}^{\mathrm{TM}}$ Real-Time PCR fluidics system according to the manufacturer's guidelines and performed by the Ramaciotti Centre for Genomics (University of New South Wales, Randwick, Australia). Amplicon libraries were pooled and a single MPS run was performed on a MiSeq platform using Miseq Control Software (MCS) version 2.4.1 (Illumina Inc., San Siego, CA, USA).

Sequencing data was received in FASTQ file format. FASTQ files were aligned to the human genome (hg19) using Burrows-Wheeler Aligner (BWA) and variant calling was performed using the Genome Analysis Toolkit (GATK), according to GATK best practice (https://www.broadinstitute.org/gatk/guide/best-practices). Annotation of variant calls was performed using ANNOVAR, version $2013 \mathrm{Ju}{ }^{43}$, while visualisation of data was performed with the Broad Institute's Integrative Genomics Viewer (IGV, v2.3, ww.broadinstitute.org).

Each sample summary was imported into Excel and filtered to display TP53 exonic and splice mutations, excluding mutations identified in intronic regions. Filtering criteria were applied to remove reads with a quality (QUAL) score less than 100. Mutations were further filtered based on their frequency in the 1000 Genome Database ${ }^{44}$. If a particular variant occurred at a frequency greater than $10 \%$ in this database, the mutation was deemed to be non-deleterious and excluded from our analysis. Lastly, mutations were filtered based on SIFT scores, an in silico tool for predicting functional effects of a mutation on the associated protein ${ }^{45}$. Variants predicted to be tolerated were excluded. All remaining variants were considered deleterious or did not have a SIFT score and were visualized using IGV. The allele frequency of each mutation was recorded upon verification of the mutation via IGV. Our analysis pipeline is summarised in Supplementary Fig. S5.

Sanger Sequencing to validate TP53 mutations. All samples in which a TP53 mutation was identified by MPS underwent Sanger sequencing using the identical DNA. Primers and PCR conditions used were those recommended by the International Agency for Research on Cancer (IARC) TP53 database (http://p53. iarc.fr/; Supplementary Table S5). PCR products were purified using a DNA Clean \& Concentrator ${ }^{\mathrm{TM}}$ Kit (Zymo Research Corporation, Irvine, CA, USA) and commercial sequencing undertaken by the Australian Genome Research Facility (AGRF; Sydney, NSW, Australia). TP53 variants were annotated against the human TP53 reference genomic sequence NC_000017.10 using Sequencher DNA Sequence Analysis Software version 4.9 (Gene Codes Corporation, Ann Arbor, MI, USA).

Statistical Analyses. Specificity and sensitivity of the association between p53 IHC score and the presence or absence of a TP53 mutation were analysed using the VassarStats Statistical Computation website (http://www. vassarstats.net/). Western blot and qRT-PCR data from analyses of ovarian cancer cell lines are expressed as the mean \pm S.E.M. from at least three independent experiments. Statistical significance was determined by one-way ANOVA. Kaplan-Meier survival analyses compared by the Log Rank (Mantel-Cox) test were undertaken to determine whether the type or location of TP53 mutation, or p53 staining pattern (graded as 'High', 'Intermediate' or 'Low') influenced overall survival in HGSOC. $P<0.05$ was considered statistically significant. With the exception of specificity and sensitivity calculations above, all other data analyses were undertaken using IBM SPSS software version 21.0 (SPSS Australasia Pty Ltd., Chatswood, NSW, Australia).

\section{References}

1. Oren, M. \& Rotter, V. Mutant p53 gain-of-function in cancer. Cold Spring Harb Perspect Biol 2, a001107 (2010).

2. Bieging, K. T., Mello, S. S. \& Attardi, L. D. Unravelling mechanisms of p53-mediated tumour suppression. Nat Rev Cancer 14, 359-370 (2014)

3. Kandoth, C. et al. Mutational landscape and significance across 12 major cancer types. Nature 502, 333-339 (2013).

4. Leroy, B. et al. The TP53 website: an integrative resource centre for the TP53 mutation database and TP53 mutant analysis. Nucleic Acids Res 41, D962-969 (2013).

5. Soussi, T. \& Wiman, K. G. Shaping genetic alterations in human cancer: the p53 mutation paradigm. Cancer Cell 12, 303-312 (2007).

6. Brady, C. A. \& Attardi, L. D. p53 at a glance. J Cell Sci 123, 2527-2532 (2010).

7. Belyi, V. A. et al. The origins and evolution of the p53 family of genes. Cold Spring Harb Perspect Biol 2, a001198 (2010).

8. Olivier, M., Hollstein, M. \& Hainaut, P. TP53 mutations in human cancers: origins, consequences, and clinical use. Cold Spring Harbor Perspect Biol 2, a001008 (2010).

9. Muller, P. A. \& Vousden, K. H. Mutant p53 in cancer: new functions and therapeutic opportunities. Cancer Cell 25, 304-317 (2014). 
10. Wong, R. P. et al. p53-R273H gains new function in induction of drug resistance through down-regulation of procaspase-3. Mol Cancer Ther 6, 1054-1061 (2007).

11. Blandino, G., Levine, A. J. \& Oren, M. Mutant p53 gain of function: differential effects of different p53 mutants on resistance of cultured cells to chemotherapy. Oncogene 18, 477-485 (1999).

12. Lehmann, S. et al. Targeting $\mathrm{p} 53$ in vivo: a first-in-human study with p53-targeting compound APR-246 in refractory hematologic malignancies and prostate cancer. J Clin Oncol 30, 3633-3639 (2012).

13. Blanden, A. R., Yu, X., Loh, S. N., Levine, A. J. \& Carpizo, D. R. Reactivating mutant p53 using small molecules as zinc metallochaperones: awakening a sleeping giant in cancer. Drug Discov Today 20, 1391-1397(2015).

14. Chen, G. X. et al. Clinical utility of recombinant adenoviral human p53 gene therapy: current perspectives. Onco Targets Ther 7 , 1901-1909 (2014).

15. Torre, L. A. et al. Global cancer statistics, 2012. CA Cancer J Clin 65, 87-108 (2015).

16. Visintin, I. et al. Diagnostic markers for early detection of ovarian cancer. Clin Cancer Res 14, 1065-1072 (2008).

17. Beesley, V. L. et al. Quality of life and treatment response among women with platinum-resistant versus platinum-sensitive ovarian cancer treated for progression: a prospective analysis. Gynecol Oncol 132, 130-136 (2014).

18. Bell, D. et al. Integrated genomic analyses of ovarian carcinoma. Nature 474, 609-615 (2011).

19. Patch, A. M. et al. Whole-genome characterization of chemoresistant ovarian cancer. Nature 521, 489-494 (2015).

20. Vang, R. et al. Molecular Alterations of TP53 are a Defining Feature of Ovarian High-Grade Serous Carcinoma: A Rereview of Cases Lacking TP53 Mutations in The Cancer Genome Atlas Ovarian Study. Int J Gynecol Pathol 35, 48-55 (2016).

21. Yemelyanova, A. et al. Immunohistochemical staining patterns of p53 can serve as a surrogate marker for TP53 mutations in ovarian carcinoma: an immunohistochemical and nucleotide sequencing analysis. Mod Pathol 24, 1248-1253 (2011).

22. Laframboise, S., Chapman, W., McLaughlin, J. \& Andrulis, I. L. p53 mutations in epithelial ovarian cancers: possible role in predicting chemoresistance. Cancer J 6, 302-308 (2000).

23. Hall, J., Paul, J. \& Brown, R. Critical evaluation of p53 as a prognostic marker in ovarian cancer. Expert Rev Mol Med 6, 1-20 (2004).

24. Wojnarowicz, P. M. et al. The genomic landscape of TP53 and p53 annotated high grade ovarian serous carcinomas from a defined founder population associated with patient outcome. PLos One 7, e45484 (2012).

25. Bernardini, M. Q. et al. Expression signatures of TP53 mutations in serous ovarian cancers. BMC Cancer 10, 237 (2010).

26. Piek, J. M. et al. Dysplastic changes in prophylactically removed Fallopian tubes of women predisposed to developing ovarian cancer. The Journal of pathology 195, 451-456 (2001).

27. Lee, Y. et al. A candidate precursor to serous carcinoma that originates in the distal fallopian tube. J Pathol 211, 26-35 (2007).

28. Carlson, J. W. et al. Serous tubal intraepithelial carcinoma: its potential role in primary peritoneal serous carcinoma and serous cancer prevention. J Clin Oncology 26, 4160-4165 (2008).

29. Ahmed, A. A. et al. Driver mutations in TP53 are ubiquitous in high grade serous carcinoma of the ovary. J Pathol 221, 49-56 (2010).

30. Havrilesky, L. et al. Prognostic significance of p53 mutation and p53 overexpression in advanced epithelial ovarian cancer: a Gynecologic Oncology Group Study. J Clin Oncol 21, 3814-3825 (2003).

31. Lassus, H., Leminen, A., Lundin, J., Lehtovirta, P. \& Butzow, R. Distinct subtypes of serous ovarian carcinoma identified by p53 determination. Gynecol Oncol 91, 504-512 (2003).

32. Kobel, M. et al. The biological and clinical value of p53 expression in pelvic high-grade serous carcinomas. J Pathol 222, 191-198 (2010).

33. Moskalev, E. A. et al. Increased detection rates of EGFR and KRAS mutations in NSCLC specimens with low tumour cell content by 454 deep sequencing. Virchows Arch 462, 409-419 (2013).

34. Lin, M. T. et al. Clinical validation of KRAS, BRAF, and EGFR mutation detection using next-generation sequencing. Am J Clin Pathol 141, 856-866 (2014).

35. de Graeff, P. et al. Modest effect of p53, EGFR and HER-2/neu on prognosis in epithelial ovarian cancer: a meta-analysis. Br J Cancer 101, 149-159 (2009).

36. Hogdall, E. V. et al. Distribution of p53 expression in tissue from 774 Danish ovarian tumour patients and its prognostic significance in ovarian carcinomas. APMIS 116, 400-409 (2008).

37. Wen, W. H. et al. p53 mutations and expression in ovarian cancers: correlation with overall survival. Int J Gynecol Pathol 18, 29-41 (1999).

38. Domcke, S., Sinha, R., Levine, D. A., Sander, C. \& Schultz, N. Evaluating cell lines as tumour models by comparison of genomic profiles. Nature Commun 4, 2126 (2013).

39. Ince, T. A. et al. Characterization of twenty-five ovarian tumour cell lines that phenocopy primary tumours. Nature Commun 6, 7419 (2015)

40. Duffy, M. J. et al. p53 as a target for the treatment of cancer. Cancer Treat Rev 40, 1153-1160 (2014).

41. Conover, C. A. et al. Biological characterization of human epithelial ovarian carcinoma cells in primary culture: the insulin-like growth factor system. Exp Cell Res 238, 439-449 (1998).

42. Kan, C. W. et al. Elevated levels of circulating microRNA-200 family members correlate with serous epithelial ovarian cancer. $B M C$ Cancer 12, 627 (2012)

43. Wang, K., Li, M. \& Hakonarson, H. ANNOVAR: functional annotation of genetic variants from high-throughput sequencing data. Nucleic Acids Res 38, e164 (2010).

44. Nielsen, R. Genomics: In search of rare human variants. Nature 467, 1050-1051 (2010).

45. Ng, P. C. \& Henikoff, S. Predicting deleterious amino acid substitutions. Genome Res 11, 863-874 (2001).

\section{Acknowledgements}

This project was supported by a Research Scholar Award from the Sydney Vital Translational Cancer Research Centre, Cancer Institute NSW [to AJC] and project grant from the Cancer Council NSW [RG13-10; to DJM]. AJC was supported by an Australian Postgraduate Award and Northern Clinical School Top-Up Scholarship. DJM was supported by the Australian Research Council (ARC) (ARC Future Fellowship [FT100100489]) and National Health and Medical Research Council (NHMRC) (NHMRC Senior Research Fellowship [APP1004799]). The Kolling Institute Gynecological Tumour Bank is thanked for supply of ovarian tumours. Mr Graham Wilkins is acknowledged for preparing hematoxylin and eosin slides and Ms Sue Smith is thanked for her assistance in preparation of cell line blocks for immunohistochemistry.

\section{Author Contributions}

A.J.C., R.C.-B. and D.J.M. conceived the experiments; A.J.C., K.-A.D. and A.C. conducted the experiments; A.J.C., T.D., A.J.G., Y.Z. and D.J.M. analysed the results; G.B.G., J.M. and S.V. collected specimens and provided patient information; A.J.C. and D.J.M. wrote the manuscript. All authors reviewed the manuscript. 


\section{Additional Information}

Supplementary information accompanies this paper at http://www.nature.com/srep

Competing financial interests: The authors declare no competing financial interests.

How to cite this article: Cole, A. J. et al. Assessing mutant p53 in primary high-grade serous ovarian cancer using immunohistochemistry and massively parallel sequencing. Sci. Rep. 6, 26191; doi: 10.1038/srep26191 (2016).

(c) (i) This work is licensed under a Creative Commons Attribution 4.0 International License. The images

or other third party material in this article are included in the article's Creative Commons license, unless indicated otherwise in the credit line; if the material is not included under the Creative Commons license, users will need to obtain permission from the license holder to reproduce the material. To view a copy of this license, visit http://creativecommons.org/licenses/by/4.0/ 